\title{
Primary Stent Retrieval for Acute Intracranial Large Artery Occlusion Due to Atherosclerotic Disease
}

\author{
Jin Soo Lee, ${ }^{\mathrm{a}}$ Ji Man Hong, ${ }^{\mathrm{a}}$ Kyu Sun Lee, ${ }^{\mathrm{a}}$ Hong Il Suh, ${ }^{\mathrm{a}}$ Jin Wook Choi, ${ }^{\mathrm{b}}$ Sun Yong Kim ${ }^{\mathrm{b}}$ \\ Departments of ${ }^{\mathrm{a}}$ Neurology, ${ }^{\mathrm{b}}$ Radiology, School of Medicine, Ajou University Medical Center, Suwon, Korea
}

Background and Purpose The goal of stent retriever-based thrombectomy is removal of embolic clots in patients with intracranial large artery occlusion. However, outcomes of stent retrieval may differ between acute arterial occlusions due to intracranial atherosclerotic disease (IAD) and those due to embolism. This case series describes the outcomes of stent retriever-based thrombectomy and rescue treatments in 9 patients with IAD-related occlusion.

Methods Among patients who underwent endovascular treatment for acute intracranial large artery occlusion, those in whom stent retrieval was attempted as first-line treatment were included in this review. IAD was defined as significant fixed focal stenosis at the occlusion site, which was evident on final angiographic assessment or observed during endovascular treatment.

Results Median number of stent retriever passes was 2 (range, 1-3), and temporary bypass was seen in all patients. Immediate partial recanalization (arterial occlusive lesion grade 2-3) was observed in 7 patients. Immediate modified thrombolysis in cerebral infarction grade 2b-3 was seen in 6 patients, but the lesions often required rescue treatment due to reocclusion or flow insufficiency. In terms of rescue treatments, angioplasty and intra-arterial tirofiban infusion seemed to be effective.

Conclusions Our findings suggest that stent retrieval can effectively remove thrombi from stenotic lesions and achieve partial recanalization despite the tendency toward reocclusion in most patients with IAD-related occlusion. Further research into the use of rescue treatments, such as tirofiban infusion and angioplasty, is warranted.

Keywords Cerebral infarction; Thrombectomy; Mechanical thrombolysis; Intracranial arteriosclerosis; Intracranial embolism and thrombosis
Correspondence: Jin Soo Lee Department of Neurology, Ajou University School of Medicine, Ajou University Hospital, 164 Worldcup-ro, Yeongtong-gu, Suwon 16499, Korea Tel: $+82-31-219-5175$ Fax: +82-31-219-5178 E-mail: jinsoo22@gmail.com

Received: July 27, 2015

Revised: August 28, 2015

Accepted: September 7, 2015

This research was partly supported by Basic Science Research Program through the National Research Foundation of Korea (NRF) funded by the Ministry of Education (NRF-2014R1A1A1008249).

The authors have no financial conflicts of interest.

\section{Introduction}

Intravenous recombinant tissue plasminogen activator (rtPA) infusion was the first approved treatment for acute ischemic stroke. ${ }^{1,2}$ However, this treatment has limited efficacy in patients with intracranial large artery occlusion, and only a subset of patients experience recanalization. ${ }^{3}$ In patients with underlying intracranial atherosclerotic disease (IAD), stenotic lesions tend to reocclude despite recanalization by intravenous rtPA. ${ }^{4}$ Notably, $\mathrm{IAD}$ is more common in Asian populations than in Western populations. ${ }^{5}$

Recently, randomized controlled studies of endovascular treatments for acute ischemic stroke due to intracranial large artery occlusion have demonstrated improved efficacy. ${ }^{6-10}$ One important factor in their success is the use of stent retrievers to remove clots. Stent retrievers were designed for thrombus, which 
can incorporate into the inner space of the stent. In situ thrombosis may occur when intracranial large artery occlusion is associated with IAD, ${ }^{11}$ but it has not been evaluated whether such occlusions can be removed via stent retrieval. Additionally, the underlying stenosis cannot be solved with stent retrieval and reocclusion can often occur, so other rescue treatments may be required. $^{12}$

There are few reports of emergent thrombectomy using stent retrievers in patients with acute ischemic stroke and IAD-related occlusion. ${ }^{13}$ In the current study, a consecutive case series is presented, with a focus on procedural factors. In addition, several types of rescue treatment are introduced.

\section{Methods}

Patients who underwent endovascular treatment for acute ischemic stroke due to intracranial large artery occlusion at a Korean university hospital between July 2013 and May 2015 were screened. Only those who underwent endovascular treatment with the Solitaire flow restoration (FR) revascularization device (Covidien, Irvine, CA, USA) as the first-line method were included. IAD was defined as significant fixed focal stenosis at the occlusion site, which was evident on final angiographic assessment or observed during endovascular treatment. Significant stenosis was defined as (1) degree of fixed stenosis $>70 \%$, or (2) any degree of fixed stenosis with either flow and perfusion impairment on angiography or an evident tendency toward reocclusion even after sufficient treatment with stent retrieval. When angioplasty or stent insertion achieved full recanalization of intracranial stenosis, the case was classified as IAD. Patients whose final angiographic assessment showed complete recanalization with arterial occlusive lesion (AOL) grade 3 were excluded from this study because their stroke etiology was proved to involve embolism. Patients with an arterial occlusion etiology of moyamoya disease, dissection, or vasculitis also were excluded. Cases with vessel injury accompanied by an interventional procedure, and those in which the occlusion site was never recanalized because the underlying IAD could not be evaluated also were excluded from this study.

Patient outcome was analyzed according to the immediate effects of stent retrieval as the primary treatment method. Modified thrombolysis in cerebral infarction grade was used to evaluate reperfusion status. ${ }^{14} \mathrm{AOL}$ grade was used to evaluate recanalization status of the occlusion site. ${ }^{14}$ Both of the above scales do not always correlate with each other. National Institutes of Health Stroke Scale score was used to evaluate neurologic severity on admission and at discharge. Modified Rankin Scale score was used to evaluate disability at 3 months. Procedural factors including operation duration, rescue treatment methods, and complications also were recorded.

\section{Results}

\section{Patients and revascularization treatments}

Among 53 patients who underwent endovascular treatment with the Solitaire FR device as the first-line method, IAD-related occlusion was observed in 9 ( 8 men, 1 woman; median age, 63 years; range, 57-77 years) (Table 1 ). Three lesions were observed to affect posterior circulation ( 2 in the basilar artery [BA] trunk, 1 in the vertebral artery), while the other lesions affected anterior circulation (all in the M1 segment of the middle cerebral artery [MCA] ). None of the lesions had any cardioembolic source. Six patients underwent intravenous rtPA infusion prior to endovascular treatment. A stent retriever was the primary device for endovascular treatment, and the Solitaire FR device was applied in all cases. A balloon guide catheter was used concomitantly, which was inflated in all cases with anterior circulation occlusion.

Table 1. Baseline characteristics and immediate effects of stent retrieval

\begin{tabular}{|c|c|c|c|c|c|c|c|c|c|c|c|}
\hline Case No. & Sex & $\begin{array}{l}\text { Age } \\
\text { (year) }\end{array}$ & $\begin{array}{c}\text { Cardioembolic } \\
\text { potential }\end{array}$ & $\begin{array}{l}\text { NIHSS score } \\
\text { on admission }\end{array}$ & $\begin{array}{c}\text { Occlusion site } \\
\text { on CTA }\end{array}$ & IV rtPA & $\begin{array}{l}\text { No. of } \\
\text { passes }\end{array}$ & $\begin{array}{c}\text { Temporary } \\
\text { bypass }\end{array}$ & $\begin{array}{l}\text { Immediate } \\
\text { mTICl grade }\end{array}$ & $\begin{array}{l}\text { Immediate } \\
\text { AOL grade }\end{array}$ & $\begin{array}{l}\text { Thrombus } \\
\text { removal }\end{array}$ \\
\hline 1 & M & 61 & None & 4 & VA & Yes & 2 & Yes & 0 & 0 & Yes* \\
\hline 2 & M & 73 & None & 18 & M1 of MCA & Yes & 1 & Yes & 3 & 2 & Yes \\
\hline 3 & M & 56 & None & 9 & M1 of MCA & Yes & 3 & Yes & 3 & 2 & Yes \\
\hline 4 & $M$ & 57 & None & 30 & BA trunk & No & 3 & Yes & $2 a$ & 1 & Yes* \\
\hline 5 & M & 57 & None & 28 & BA trunk & Yes & 2 & Yes & 3 & 2 & Yes \\
\hline 6 & M & 63 & None & 18 & M1 of MCA & No & 2 & Yes & $2 b$ & 2 & Yes \\
\hline 7 & $\mathrm{~F}$ & 83 & None & 17 & M1 of MCA & No & 1 & Yes & 3 & 2 & Yes \\
\hline 8 & M & 81 & None & 16 & $\mathrm{M} 1$ of MCA & Yes & 1 & Yes & 3 & 1 & Yes \\
\hline 9 & M & 68 & None & 9 & M1 of MCA & Yes & 2 & Yes & 3 & 2 & Yes \\
\hline
\end{tabular}

*New distal artery occlusion was seen after stent retrieval was attempted.

AOL, arterial occlusive lesion; BA, basilar artery; CTA, computed tomography angiography; IV, intravenous; MCA, middle cerebral artery; mTICl, modified thrombolysis in cerebral infarction; NIHSS, National Institutes of Health Stroke Scale; rtPA, recombinant tissue plasminogen activator; VA, vertebral artery. 
Table 2. Rescue treatments and procedure times

\begin{tabular}{|c|c|c|c|c|c|c|c|}
\hline Case No. & Rescue therapy & Angioplasty & Stenting & Tirofiban & $\begin{array}{l}\text { Onset to puncture } \\
\text { (minute) }\end{array}$ & $\begin{array}{l}\text { Puncture to } 1 \text { st deployment } \\
\text { (minute) }\end{array}$ & $\begin{array}{l}\text { 1st deployment to recanalization } \\
\text { (minute) }\end{array}$ \\
\hline 1 & Yes & Yes & - & - & 173 & 53 & 48 \\
\hline 2 & Yes & Yes & - & - & 381 & 68 & 29 \\
\hline 3 & Yes & Yes & - & - & 287 & 33 & 69 \\
\hline 4 & Yes & Yes & - & - & 392 & 39 & 59 \\
\hline 5 & Yes & Yes & Yes & - & 203 & 66 & 84 \\
\hline 6 & No & - & - & - & 352 & 29 & 33 \\
\hline 7 & Yes & - & - & Yes & $698^{*}$ & 30 & 48 \\
\hline 8 & Yes & - & - & Yes & 154 & 40 & 38 \\
\hline 9 & No & - & - & - & 313 & 31 & 31 \\
\hline
\end{tabular}

*Patient had wake-up stroke; therefore, onset time was last normal time before sleep. Endovascular treatment was determined by institutional diffusion-perfusion mismatch criteria.

Table 3. Procedural and clinical outcomes after endovascular treatment

\begin{tabular}{|c|c|c|c|c|c|c|c|c|}
\hline Case No. & $\begin{array}{l}\text { Final } \mathrm{AOL} \\
\text { grade }\end{array}$ & $\begin{array}{l}\text { Final } m \mathrm{mICl} \\
\text { grade }\end{array}$ & $\mathrm{ICH}$ & SAH & Dissection & $\begin{array}{c}\text { Stenotic degree on CTA } \\
\text { within } 10 \text { days }(\%)\end{array}$ & $\begin{array}{c}\text { NIHSS score at } \\
\text { discharge }\end{array}$ & $\begin{array}{l}\text { mRS score at } \\
3 \text { months }\end{array}$ \\
\hline 1 & 2 & $2 b$ & - & - & - & 40 & 0 & 0 \\
\hline 2 & 2 & $2 b$ & - & - & - & 20 & 16 & 5 \\
\hline 3 & 2 & 3 & - & - & - & 80 & 3 & 1 \\
\hline 4 & 2 & $2 a$ & - & - & - & 100 & 30 & 5 \\
\hline 5 & 2 & $2 b$ & - & - & - & 0 & 28 & 5 \\
\hline 6 & 2 & $2 b$ & - & - & - & 70 & 10 & 4 \\
\hline 7 & 2 & 3 & - & - & - & 90 & 1 & 1 \\
\hline 8 & 2 & 3 & - & - & - & 90 & 8 & 1 \\
\hline 9 & 2 & 3 & - & - & - & $100^{*}$ & 6 & 1 \\
\hline
\end{tabular}

*Neurologic deterioration and reocclusion on repeat CTA were observed 5 hours after the emergent interventional procedure. The second immediate procedure including angioplasty was successful.

AOL, arterial occlusive lesion; CTA, computed tomography angiography; ICH, intracerebral hemorrhage; mRS, modified Rankin Scale; mTICI, modified thrombolysis in cerebral infarction; NIHSS, National Institutes of Health Stroke Scale; SAH, subarachnoid hemorrhage.

\section{Immediate effects of stent retrieval and rescue treatments}

Median number of stent retriever passes was 2 (range, 1-3). Temporary bypass was seen in all patients when the stent retriever was deployed. Following thrombectomy, immediate reperfusion (modified thrombolysis in cerebral infarction grade 2b-3) was seen in 7 patients, and immediate partial recanalization (AOL grade 2-3) was seen in 6. On angiography, the thrombi were somewhat removed in all patients; however, most removed clots were very small or not visible in the stent. Table 2 shows the rescue treatment types and methods, while Table 3 shows the procedural and clinical outcomes. Angioplasty was the most commonly used rescue treatment (in 5 patients) (Figure 1), while intra-arterial tirofiban infusion was performed in 2 patients. Two patients did not undergo immediate rescue treatment; however, one patient (patient No. 9) required a second emergent procedure after showing severe neurologic deterioration 5 hours after the first procedure. He underwent angioplasty and tirofiban infusion, which resulted in successful revascularization and improved modified Rankin Scale score.

\section{Outcomes of patients with BA trunk occlusion}

Both patients with IAD-related occlusion affecting the $\mathrm{BA}$ trunk had very poor outcomes. In both cases, the stenotic lengths were $>10 \mathrm{~mm}$. In patient No. 4, the lesion was repeatedly reoccluded despite several stent retrieval trials and partial recanalization after angioplasty. Computed tomography $(\mathrm{CT})$ angiography showed that the BA was reoccluded 5 hours later, and a second emergent procedure was performed. The patient's consciousness level improved, but he still demonstrated locked-in syndrome 3 months after presentation. In patient No. 5, the lesion was repeatedly reoccluded during stent retrieval trials, so successive angioplasty was performed. Despite those efforts, blood flow remained stagnant at the stenotic site. Thus, a Wingspan stent (Stryker, Kalamazoo, MI, USA) was deployed, and poststenting angioplasty was performed. Although the patient's recanalization and reperfusion grades improved, as did his consciousness level, he remained locked-in at 3 months. 

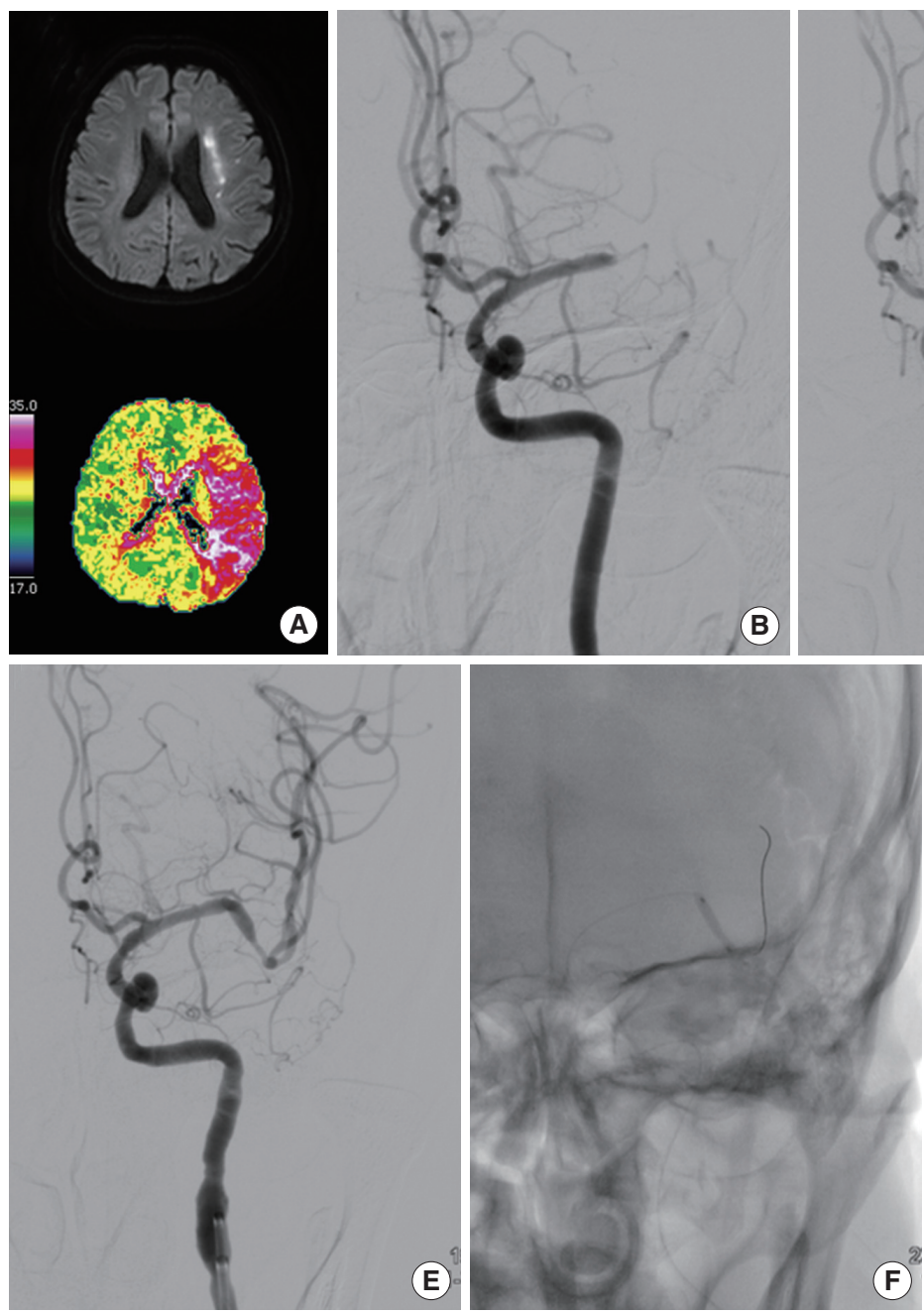
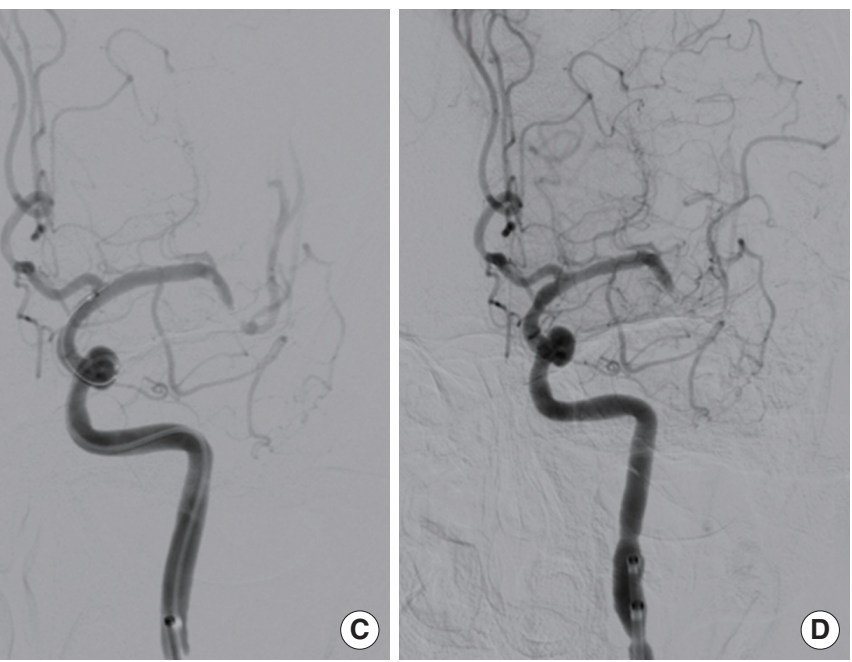

(D)

Figure 1. A 56-year-old man (patient No. 3) presented with left hemispheric syndrome. Baseline magnetic resonance imaging (MRI) shows an acute left internal borderzone infarction on diffusion-weighted imaging, and a large perfusion defect on time-to-peak of perfusion-weighted imaging (A). Initial cerebral angiography shows occlusion of the distal M1 segment of the left middle cerebral artery (B). Temporary blood flow bypass is seen when the Solitaire FR stent is being deployed (C). Although some clots were removed at the occlusion site, occlusion is still seen at a more distal vessel (D). After 3 stent retrieval passes, partial recanalization was achieved, but flow still seems stagnated (E). After 2 angioplasties (F), complete reperfusion was fully achieved (G). Repeat MRI performed on the fifth day of admission shows similar infarct volume and complete recovery of the perfusion defect $(\mathrm{H})$. The patient's modified Rankin Scale score was 1 at 3 months and 0 at 1 year.

\section{Discussion}

In this study, we found that the effectiveness of stent retrievers for treating acute IAD-related occlusion was somewhat limited because of hidden stenosis. Despite this limited effectiveness, in situ thrombi could be removed well, and partial revascularization was achieved in most cases. These findings demonstrate that stent retrieval could work well as the primary endovascular treatment. Furthermore, degree and length of stenosis could be determined upon stent retriever deployment, which was helpful in the planning of subsequent rescue treatments.

The performance of stent retrievers for treating IAD-related occlusion, and even its frequency, has been rarely reported. In this study, we tried to describe the immediate effects of endo- vascular treatment in 9 patients. The proportion of IAD-related occlusion at our institution appears to be higher than that reported for non-Asian populations. Indeed, one Korean study showed that IAD-related occlusion accounted for $23 \%$ of acute ischemic stroke cases and endovascular treatment. ${ }^{15}$ In the current study, patients who had acute ischemic stroke due to intracranial large artery occlusion underwent endovascular treatment, and IAD-related occlusion was confirmed by transfemoral cerebral angiography in $17 \%$ of the cohort. As a comparison, in a French study, just $5.5 \%$ of patients treated with stent retrieval showed intracranial stenosis. 13 Nevertheless, the findings regarding frequency should be interpreted with caution because these studies were not performed with prospective enrollment criteria. In addition, the definition of IAD might differ 
somewhat among studies.

IAD-related occlusion may result from in situ thrombosis in a stenotic lesion. When an inflammatory response occurs in unstable atherosclerotic plaque, platelets aggregate into a thrombus and ultimately occlude the vessel. ${ }^{16}$ Considering this, stent retriever-based thrombectomy is an appropriate treatment choice, and its effectiveness has been demonstrated in the present series. Temporary bypass or partial recanalization generally was achieved with the first pass. All patients showed angiographic evidence of thrombus reduction or partial recanalization. Thus, the stent retriever was shown to be effective for thrombus removal, though the retrieved thrombi were generally small. On the other hand, an early bypass effect is important to improve patient outcomes because blood supply can be restored more rapidly to the ischemic brain. ${ }^{17}$ Therefore, stent retrievers could be considered as a primary treatment option for patients with $\mathrm{IAD}$-related occlusion who require emergent endovascular treatment.

Stent retrievers are also useful for planning rescue treatments (e.g., angioplasty or additional stenting) because IAD-related occlusions tend to reocclude. When a stent retriever is deployed and bypass flow is observed, interventionalists can measure the stenotic length and determine the curvature of the vessel. Different rescue treatments were not tested in the current study.

Angioplasty was effective in the distal MCA and intracranial vertebral arteries. Intracranial stenting, however, was not performed routinely due to concerns of hemorrhagic transformation, which cannot be treated with antithrombotics. In addition, angioplasty with or without stenting should be decided with caution when performed on a stenotic vessel near its branching small arteries. In one patient who had severe BA trunk occlusion, angioplasty was performed and a Wingspan stent was deployed following repeat stent retriever-based thrombectomy. The artery achieved complete recanalization and was patent on followup CT angiography. Unfortunately, although the patient's level of consciousness recovered, he remained in a locked-in state due to a diffuse pontine lesion. If perforators exist near the stenotic lesion (e.g., anterior inferior cerebellar arteries in the BA trunk or striatocapsular arteries in the MCA trunk), angioplasty and intracranial stenting should be performed carefully, because ischemic complications have been reported to occur more often in the $\mathrm{BA}$, according to a previous study regarding intracranial stenting for intracranial stenosis. ${ }^{18}$

The effect of intra-arterial tirofiban infusion in this study is consistent with that reported in a previous study, ${ }^{12}$ and it did not cause unwanted hemorrhagic complications even on the basis of intravenous full-dose rtPA in our series. Presence of stenosis itself is not a casual factor for stroke, but inflammation and platelet aggregation can lead to an ischemic event. ${ }^{16}$ Tirofiban is a GPIIb/IIIa antagonist with a short half-life, so its effect is rapid. In some of our patients, tirofiban could stabilize the inflamed stenotic lesion and maintain blood flow, even without further recanalization procedures, such as angioplasty. Despite our results, randomized multicenter trials are necessary to determine the optimal rescue treatments following stent retrieverbased thrombectomy.

This study has some limitations. First, it was a retrospective study performed at one hospital. In addition to the rare disease entity, the small cohort requires caution when interpreting the results. Nevertheless, this is the first study to describe the performance of stent retriever-based thrombectomy for intracranial large artery occlusion due to IAD. Another limitation is the possibility of selection error. For example, we excluded patients whose vessels were never recanalized. Since IAD may be hidden in those cases, the recanalization power might have been overestimated. Consequently, we plan to perform a further prospective multicenter study.

\section{Conclusions}

IAD-related occlusion was observed in $17 \%$ of patients who underwent stent retriever-based thrombectomy. This method successfully removed in situ thrombi and helped achieve immediate partial recanalization in most cases. While several types of rescue treatment are possible after stent retrieval, further multicenter trials are needed to identify the optimal methods.

\section{References}

1. The National Institute of Neurological Disorders and Stroke rtPA Stroke Study Group. Tissue plasminogen activator for acute ischemic stroke. N Engl J Med 1995;333:1581-1587.

2. Hacke W, Kaste M, Bluhmki E, Brozman M, Davalos A, Guidetti D, et al. Thrombolysis with alteplase 3 to 4.5 hours after acute ischemic stroke. N Engl J Med 2008;359:1317-1329.

3. Bhatia R, Hill MD, Shobha N, Menon B, Bal S, Kochar P, et al. Low rates of acute recanalization with intravenous recombinant tissue plasminogen activator in ischemic stroke: Real-world experience and a call for action. Stroke 2010;41:2254-2258.

4. Heo JH, Lee KY, Kim SH, Kim DI. Immediate reocclusion following a successful thrombolysis in acute stroke: a pilot study. Neurology 2003;60:1684-1687.

5. Kim JS, Bonovich D. Research on intracranial atherosclerosis from the east and west: why are the results different? J Stroke 2014;16:105-113.

6. Berkhemer OA, Fransen PS, Beumer D, van den Berg LA, 
Lingsma HF, Yoo AJ, et al. A randomized trial of intraarterial treatment for acute ischemic stroke. N Engl J Med 2015;372: 11-20.

7. Goyal M, Demchuk AM, Menon BK, Eesa M, Rempel JL, Thornton J, et al. Randomized assessment of rapid endovascular treatment of ischemic stroke. NEngl J Med 2015;372:1019-1030.

8. Saver JL, Goyal M, Bonafe A, Diener HC, Levy EI, Pereira VM, et al. Stent-retriever thrombectomy after intravenous t-pa vs. T-pa alone in stroke. N Engl J Med 2015;372:2285-2295.

9. Campbell BC, Mitchell PJ, Kleinig TJ, Dewey HM, Churilov L, Yassi N, et al. Endovascular therapy for ischemic stroke with perfusion-imaging selection. NEngl JMed 2015;372:1009-1018.

10. Jovin TG, Chamorro A, Cobo E, de Miquel MA, Molina CA, Rovira A, et al. Thrombectomy within 8 hours after symptom onset in ischemic stroke. N Engl J Med 2015;372:2296-2306.

11. Bang OY. Intracranial atherosclerosis: Current understanding and perspectives. J Stroke 2014;16:27-35.

12. Kang DH, Kim YW, Hwang YH, Park SP, Kim YS, Baik SK. Instant reocclusion following mechanical thrombectomy of in situ thromboocclusion and the role of low-dose intra-arterial tirofiban. Cerebrovasc Dis 2014;37:350-355.

13. Gascou G, Lobotesis K, Machi P, Maldonado I, Vendrell JF,
Riquelme C, et al. Stent retrievers in acute ischemic stroke: complications and failures during the perioperative period. AJNR Am JNeuroradiol 2014;35:734-740.

14. Zaidat OO, Yoo AJ, Khatri P, Tomsick TA, von Kummer R, Saver JL, et al. Recommendations on angiographic revascularization grading standards for acute ischemic stroke: a consensus statement. Stroke 2013;44:2650-2663.

15. Yoon W, Kim SK, Park MS, Kim BC, Kang HK. Endovascular treatment and the outcomes of atherosclerotic intracranial stenosis in patients with hyperacute stroke. Neurosurgery 2015; 76:680-686.

16. Libby P. Inflammation in atherosclerosis. Nature 2002;420: 868-874.

17. Roth C, Papanagiotou P, Behnke S, Walter S, Haass A, Becker $\mathrm{C}$, et al. Stent-assisted mechanical recanalization for treatment of acute intracerebral artery occlusions. Stroke 2010;41:25592567.

18. Fiorella D, Derdeyn CP, Lynn MJ, Barnwell SL, Hoh BL, Levy EI, et al. Detailed analysis of periprocedural strokes in patients undergoing intracranial stenting in stenting and aggressive medical management for preventing recurrent stroke in intracranial stenosis (SAMMPRIS). Stroke 2012;43:2682-2688. 